\title{
Triple-negative breast cancer with brain metastases: a comparison between basal-like and non-basal-like biological subtypes
}

\author{
Anna Niwińska • Wojciech Olszewski • \\ Magdalena Murawska $\cdot$ Katarzyna Pogoda
}

Received: 16 November 2010/Accepted: 22 May 2011/Published online: 9 June 2011

(c) The Author(s) 2011. This article is published with open access at Springerlink.com

\begin{abstract}
The aim of this study was to divide the group of triple-negative breast cancer patients with brain metastases into basal-like and non-basal-like biological subtypes in order to compare clinical features and survival rates in those two groups. A comprehensive analysis of 111 consecutive triple-negative breast cancer patients with brain metastases treated in the years 2003-2009 was performed. In 75 patients, immunohistochemistry was used as a surrogate of microarray in order to evaluate the expression of three basal markers: cytokeratin 5/6 (CK 5/6), EGFR/ HER1 and c-KIT. The basal-like (ER/PgR/HER2-negative, CK5/6positive and/or HER1-positive) and non-basal-like (ER/PgR/HER2-negative, CK5/6-negative, HER1-negative) subsets were selected. Clinical features and survivals were compared in both groups. In the group of 111 triplenegative breast cancer patients, median DFS, OS and survival from brain metastases were 20, 29 and 4 months, respectively. In 75 patients who were evaluable for basal markers, median DFS, OS and survival from brain metastases were 18, 26 and 3.2 months, respectively. In the basal-like subtype, the survival rates were 15, 26 and 3 months, respectively, and in the non-basal-like subtypes,
\end{abstract}

\footnotetext{
A. Niwińska $(\bowtie) \cdot$ K. Pogoda

Department of Breast Cancer and Reconstructive Surgery,

The Maria Skłodowska-Curie Memorial Cancer Center and Institute of Oncology, ul. Roentgena 5, 02-781 Warsaw, Poland e-mail: alphaonetau@poczta.onet.pl

W. Olszewski

Department of Pathology, The Maria Skłodowska-Curie Memorial Cancer Center and Institute of Oncology, Warsaw, Poland

M. Murawska

Department of Biostatistics, Erasmus University Medical Center, Rotterdam, Netherlands
}

they were 20, 30 and 2.8 months, respectively. No statistically significant differences in survivals were detected between the basal-like and non-basal-like biological subtypes. Factors influencing survival from brain metastases were: Karnofsky performance status (KPS), the status of extracranial disease and age. Biological markers differentiating triple-negative group into basal-like and non-basallike subtype (CK 5/6, HER1, c-KIT) had no influence on survival. In patients with triple-negative breast cancer and brain metastases, well-known clinical, but not molecular, features correlated with survival.

Keywords Basal-like breast cancer - Brain metastases . Cytokeratin 5/6 · EGFR/HER1 receptor · Triple-negative breast cancer

\section{Introduction}

Triple-negative breast cancer represents a distinct subset of breast cancer that exhibits a more aggressive course as compared to other biological subtypes of cancer [1-7]. The aggressiveness of the disease is best illustrated by the fact that the peak risk of recurrence of the disease is between the first and third year following diagnosis, and that survival after recurrence is significantly shorter than that observed in patients with non-triple-negative controls [4, 6 , $8-10]$. Surprisingly, patients who did not have a recurrence of the disease within the first 8 years after the diagnosis did not subsequently relapse. However, in other subtypes of breast cancer, the risk of recurrence keeps rising as time progresses $[4,11]$. The recurrence pattern of triple-negative breast cancer also differs from other biological subtypes of cancer. In addition, the most characteristic sites of metastases include the brain and lungs $[3,6,12]$. 
From a biological point of view, triple-negative breast cancer remains a heterogeneous group with difficult-todefine subtypes. Gene expression studies have shown that ER-negative and HER2-negative tumors are clustered into at least three distinct molecular classes: basal-like, normallike and claudin-negative breast cancer [1, 11, 13-19]. Most of triple-negative tumors show basal-like phenotype which can be subdivided into pure variant and myoepithelial variant [20]. Many "immunohistochemical (IHC) signatures" have been described as a surrogate of microarray, but the panel proposed by Nielsen et al. [21] seems to be the best example of the classification to date. In this classification, basal-like cancers are defined as those lacking ER and HER2 expression and expressing cytokeratin 5/6 (CK 5/6) and/or Epidermal Growth Factor Receptor (EGFR or HER1). This panel has $100 \%$ specificity and $76 \%$ sensitivity for identification of basal-like cancers.

Patients with triple-negative breast cancer and brain metastases have the poorest prognosis out of all biological subtypes of breast cancer after dissemination to the brain [3]. It is not known whether such a short survival depends on clinical features (performance status, dissemination of the disease to other organs), or the affiliation to the specific biological subset, which can be selected by molecular markers, for example basal cytokeratins.

The aim of the present study was to analyze clinical features and survival of triple-negative breast cancer patients with brain metastases and to compare basal-like and non-basal-like subtypes in order to establish the clinical value of basal biological markers differentiating these subtypes.

\section{Materials and methods}

Between 1 January 2003 and 31 December 2009, 111 patients with triple-negative (ER-negative, PgR-negative, HER2-negative) breast cancer and brain metastases were treated in the Breast Cancer and Reconstructive Surgery Department at The Maria Sklodowska-Curie Memorial Cancer Center, Warsaw, Poland. The observation of the patients started at the time of the detection of brain metastases and all data were collected prospectively in our database. In each case, treatment options were approved by a team of medical oncologists, radiation oncologist, neurologist and neurosurgeon, and were performed after patients had signed written consent. Immunohistochemistry (IHC) was carried out in order to evaluate levels of ER, PgR and HER2 expression in primary breast tumors. Staining was performed using primary antibodies against ER (Clone 6F11, Novocastra, dilution scale 1:100); against PR (Clone 16, Novocastra, dilution scale 1:200) and against HER2 (Polyclonal Hercep Test, DAKO). For the evaluation of ER and PR expression, all cases with $10 \%$ or higher stained cancer nuclei were classified as positive. Fluorescence in situ hybridization (FISH) was used for all HER2 2+ tumors using the HER2 DNA Probe Kit Abbott (Vysis). HER2-positive staining was defined as IHC 3+ or, in the case of IHC 2+, FISH-positive. HER2-negativity was defined as IHC $0,1+$ or $2+$, along with negative FISH results.

Immunohistochemistry for cytokeratin 5/6 (Clone D5/ 16B4, DAKO, dilution scale 1:100), HER1 (EGFR pharm Dx, Clone 2-18C9, DAKO, RTU) and c-KIT (CD117, Policlonal, DAKO, dilution scale 1:500), as a surrogate of cDNA microarray, was performed in 75 patients in whom paraffin blocs with primary tumor samples were available in the pathology archives. These assays were performed after the detection of brain metastases. Based on the definition by Nielsen et al. [21], tumors were divided into basal-like and non-basal-like biological subsets.

In 36 out of 111 patients, the analysis of basal markers was impossible to perform for the following reasons: firstly, there was an insufficient amount of biological material to perform detailed analysis. In some of those patients, only core biopsy or fine needle biopsy was performed to confirm the initial diagnosis of breast cancer and, after detection of brain metastases, we did not have enough biological material to perform IHC analysis. Secondly, some patients were treated in different hospitals before the dissemination of the disease, and after the detection of brain metastases, it was impossible to gain access to paraffin blocks from their primary tumors. Thirdly, according to information provided by the remaining patients, the IHC was impossible to perform due to technical difficulties that occurred during pathological processing.

Statistical analysis

Descriptive statistics were used to determine patient demographics and clinical characteristics. In order to compare categorical tumor features in the basal-like and non-basal-like subsets, the chi-square test was used. For those categorical variables in which the chi-square test was inappropriate because of small sample size, the Fisher exact test was used. A univariate analysis and Cox proportional hazards model were developed to identify factors influencing survival after brain metastasis in patients with triple-negative breast cancer. The following factors were analyzed in a Cox model: $\operatorname{KPS}(<70$ vs. $\geq 70)$, age at detection of brain metastases $(<50$ vs. $\geq 50)$, extracranial disease (absent vs. present), extracranial disease (controlled vs. uncontrolled), lung metastases (absent vs. present), brain metastases as the first or the only metastatic site 
(yes vs. no), neurosurgery (yes vs. no), systemic treatment after brain metastases (yes vs. no), basal subtype vs. nonbasal subtype. Disease-free survivals (DFS), overall survivals (OS, from initial diagnosis of breast cancer), and survivals from the detection of brain metastases in the entire group and in biological subgroups were estimated using the Kaplan-Meier method and compared using the log-rank test.

\section{Results}

Clinical characteristics of the entire group are presented in Table 1. In the group of 111 patients, histopathology and metastatic spread that is typical for triple-negative breast cancer was revealed. In most of the patients, ductal cancer with histological grade 3 was detected, although medullary and metaplastic cancers were also found. Lungs and brain were the most relevant sites of distant metastases. Among 75 patients in whom basal markers were assessed, 48 (64\%) tumors expressed CK5/6, 26 (36\%) tumors expressed HER1 and $13(17 \%)$ expressed c-KIT. In 6 tumors $(8 \%)$, expression of three basal biological markers was detected and 13 tumors (17\%) did not express any of them. Based on the definition by Nielsen et al. [21], basal-like tumors were identified based on panel of four antibodies (ER, HER2, HER1 and cytokeratin 5/6). There were 57 patients $(76 \%)$ with basal-like and 18 (24\%) with nonbasal-like biological subtypes. The comparison between basal-like and non-basal-like subsets is presented in Table 2. There were no significant differences between both groups in relation to patients' age at initial diagnosis, clinical stage and tumor histologic grade; however, medullar and metaplastic cancers were observed only in the basal-like subset. Bone metastases, liver metastases and brain metastases as a first site of dissemination were all equally distributed in both groups, but lung metastases were more common in the non-basal subtype.

DFS, OS and survival from brain metastases in triplenegative, basal-like and non-basal-like subsets are presented in Table 3. In the entire group of 111 triple-negative breast cancer patients with brain metastases, median DFS, OS and survival from brain metastases were 20, 29 and 4 months, respectively. In 75 patients with known basal markers, median DFS, OS and survival from brain metastases were 18, 26 and 3.2 months, respectively. In the basal-like subtype, they were 15, 26 and 3 months, respectively, and in the non-basal-like subtype they were 20, 30 and 2.8 months, respectively. No statistically significant differences in DFS, OS and survival from brain metastases were detected between basal-like and nonbasal-like biological subtypes.
Table 1 Characteristics of 111 patients with triple-negative breast cancer patients with brain metastases

\begin{tabular}{|c|c|c|}
\hline Characteristic & No. of patients & $\%$ \\
\hline \multicolumn{3}{|l|}{ Initial TNM stage } \\
\hline I & 18 & 16 \\
\hline II & 45 & 41 \\
\hline III & 39 & 35 \\
\hline IV & 9 & 8 \\
\hline \multicolumn{3}{|l|}{ Histological type } \\
\hline Ductal carcinoma & 78 & 70 \\
\hline Lobular carcinoma & 2 & 1 \\
\hline Medullar carcinoma & 3 & 3 \\
\hline Papillar carcinoma & 1 & 1 \\
\hline Mucinous carcinoma & 1 & 1 \\
\hline Metaplastic carcinoma & 1 & 1 \\
\hline Neuroendocrine carcinoma & 1 & 1 \\
\hline \multicolumn{3}{|l|}{ Cancer cells or invasive cancer after } \\
\hline Chemotherapy & 24 & 22 \\
\hline \multicolumn{3}{|l|}{ Number of brain metastases } \\
\hline 1 & 27 & 24 \\
\hline 2 & 7 & 6.5 \\
\hline 3 & 6 & 5.5 \\
\hline Multiple & 71 & 64 \\
\hline \multicolumn{3}{|l|}{ Localization of metastases } \\
\hline Brain as the first or only site & 36 & 32 \\
\hline Liver & 19 & 17 \\
\hline Lung & 54 & 49 \\
\hline Bone & 27 & 24 \\
\hline Locoregional recurrence & 34 & 31 \\
\hline Neurosurgery & 20 & 18 \\
\hline Systemic therapy after WBRT ${ }^{\mathrm{a}, \mathrm{b}}$ & 59 & 53 \\
\hline
\end{tabular}

${ }^{\mathrm{a}}$ WBRT whole-brain radiation therapy

${ }^{\mathrm{b}}$ In most patients, several lines of chemotherapy were used

The analysis of factors influencing survival from brain metastases in triple-negative breast cancer patients with brain metastases, including clinical and biological factors was assessed in univariate and multivariate analysis. The results of univariate analysis of the group of 111 patients are presented in Table 4 . The results of Cox multivariate analysis (final model) is presented in Table 5. Multivariate analysis revealed that clinical, and not biological factors influenced survival from brain metastases. They included KPS and the status of extracranial metastases. Patients with good performance status and those with controlled extracranial disease lived longer. Age at the detection of brain metastases was a factor of borderline significance; younger patients lived longer. None of three analyzed basal markers (CK 5/6, HER1, c-KIT) had any influence on survival from brain metastases. 
Table 2 Differences in clinical features between patients with basal-like (ER/PgR/HER2-

negative, CK5/6-positive and/or HER1-positive) and non-basallike (ER/PgR/HER2-negative, CK5/6-negative and HER1negative) biological subtypes of triple-negative breast cancer (75 patients)

Table 3 Median survival of patients with triple-negative breast cancer ( 75 patients), basal-like subtype (57 patients) and non-basal-like subtype (18 patients)

\begin{tabular}{|c|c|c|c|}
\hline Factor & $\begin{array}{l}\text { Basal-like subtype }(\%) \\
\text { (ER/PgR/HER2-negative, } \\
\text { CK5/6-positive and/or } \\
\text { HER1-positive) }\end{array}$ & $\begin{array}{l}\text { Non-basal-like subtype }(\%) \\
\text { (ER/PgR/HER2-negative, } \\
\text { CK5/6-negative and } \\
\text { HER1-negative) }\end{array}$ & $P$ value \\
\hline Number of patients & $57(76 \%)$ & $18(24 \%)$ & \\
\hline Age at initial diagnosis (years) & 50 & 47 & 0.343 \\
\hline Age at brain metastases (years) & 54 & 50 & 0.495 \\
\hline \multicolumn{4}{|l|}{ KPS } \\
\hline$<70$ & $28(49 \%)$ & $6(33 \%)$ & \\
\hline$\geq 70$ & $29(51 \%)$ & $12(67 \%)$ & 0.184 \\
\hline \multicolumn{4}{|l|}{ Initial TNM stage } \\
\hline I & $8(14 \%)$ & 0 & \\
\hline II & $20(35 \%)$ & $10(56 \%)$ & \\
\hline III & $23(40 \%)$ & $7(39 \%)$ & \\
\hline IV & $6(11 \%)$ & $1(5 \%)$ & 0.062 \\
\hline \multicolumn{4}{|l|}{ Histological type and grade } \\
\hline Ductal carcinoma Grade 3 & $30(55 \%)$ & $10(59 \%)$ & \\
\hline Other & $25(45 \%)$ & $7(41 \%)$ & 0.49 \\
\hline \multicolumn{4}{|l|}{ Number of brain metastases } \\
\hline 1 & $13(23 \%)$ & $4(22 \%)$ & \\
\hline 2 & $3(5 \%)$ & $1(6 \%)$ & \\
\hline 3 & $3(5 \%)$ & 0 & \\
\hline Multiple & $38(67 \%)$ & $13(72 \%)$ & 1.0 \\
\hline Brain as the first/only site & $20(35 \%)$ & $4(22 \%)$ & 0.308 \\
\hline Extracranial metastases & $37(65 \%)$ & $15(83 \%)$ & 0.116 \\
\hline Lung metastases & $22(39 \%)$ & $13(72 \%)$ & 0.013 \\
\hline Liver metastases & $9(16 \%)$ & $2(11 \%)$ & 0.447 \\
\hline Bone metastases & $15(26 \%)$ & $3(17 \%)$ & 0.310 \\
\hline Loca/locoregional recurrence & $14(25 \%)$ & $7(39 \%)$ & 0.200 \\
\hline Neurosurgery of brain metastasis & $12(21 \%)$ & $1(6 \%)$ & 0.120 \\
\hline Systemic treatment after WBRT & $29(51 \%)$ & $11(61 \%)$ & 0.314 \\
\hline $\mathrm{c}-\mathrm{KIT}$ & $9(16 \%)$ & $4(22 \%)$ & 0.575 \\
\hline
\end{tabular}

\begin{tabular}{lllll}
\hline & No. of patients & $\begin{array}{l}\text { Median survival } \\
\text { (months) }\end{array}$ & $95 \%$ CI & $P$-value \\
\hline $\begin{array}{l}\text { Disease-free survival } \\
\text { Triple-negative }\end{array}$ & 75 & & & \\
Basal-like & 57 & 18 & $13.728 ; 21.600$ & \\
Non-basal-like & 18 & 15 & $8.764 ; 19.308$ & \\
Overall survival & & 20 & & 0.284 \\
Triple-negative & 75 & & $17.172 ; 34.248$ & \\
Basal-like & 57 & 26 & $17.292 ; 31.428$ & \\
Non-basal-like & 18 & 24 & $19.188 ; 40.764$ & 0.227 \\
Survival from brain metastases & 30 & & \\
Triple-negative & 75 & & $2.532 ; 3.900$ & \\
Basal-like & 57 & 3.2 & $2.904 ; 3.528$ & \\
Non-basal-like & 18 & 3.2 & $0.000 ; 5.892$ & 0.880 \\
\hline
\end{tabular}


Table 4 Factors influencing survival from brain metastases in patients with triple-negative breast cancer: univariate analysis (111 patients)

\begin{tabular}{|c|c|c|}
\hline Covariate & $\begin{array}{l}\text { Median time } \\
\text { (months) }\end{array}$ & $P$ value \\
\hline \multicolumn{3}{|l|}{ KPS } \\
\hline$<70$ & 2.2 & \\
\hline$\geq 70$ & 6.3 & $<0.0001$ \\
\hline \multicolumn{3}{|l|}{ Age at initial diagnosis } \\
\hline$<50$ & 3.3 & \\
\hline $50-65$ & 3.9 & \\
\hline$>65$ & 3.6 & 0.692 \\
\hline \multicolumn{3}{|c|}{ Age at the detection of brain metastases } \\
\hline$<50$ & 6.3 & \\
\hline $50-65$ & 3.0 & \\
\hline$>65$ & 3.6 & 0.024 \\
\hline \multicolumn{3}{|l|}{ Initial TNM stage } \\
\hline $\mathrm{I}+\mathrm{II}$ & 3.3 & \\
\hline III + IV & 4.8 & 0.158 \\
\hline \multicolumn{3}{|l|}{ Extracranial disease } \\
\hline Present & 3.2 & \\
\hline Absent & 6.3 & 0.063 \\
\hline \multicolumn{3}{|l|}{ Extracranial disease } \\
\hline Controlled (stable/responsive) & 8.2 & \\
\hline Uncontrolled (progressive) & 2.8 & $<0.0001$ \\
\hline \multicolumn{3}{|l|}{ Locoregional recurrence } \\
\hline Present & 4.2 & \\
\hline Absent & 2.8 & 0.304 \\
\hline \multicolumn{3}{|l|}{ Lung metastases } \\
\hline Present & 3.1 & \\
\hline Absent & 6.3 & 0.045 \\
\hline \multicolumn{3}{|l|}{ Neurosurgery } \\
\hline Yes & 12 & \\
\hline No & 3.2 & 0.002 \\
\hline \multicolumn{3}{|l|}{ Systemic treatment after WBRT } \\
\hline Yes & 4.9 & \\
\hline No & 2.6 & 0.148 \\
\hline \multicolumn{3}{|l|}{ Type of chemotherapy after WBRT } \\
\hline With capecitabine & 3.1 & \\
\hline With vinorelbine & 5.9 & \\
\hline With taxanes & 5.4 & \\
\hline With antracyclines & 9.8 & \\
\hline With platinum salts or etoposide & 3.9 & \\
\hline Without chemotherapy & 2.6 & 0.496 \\
\hline
\end{tabular}

\section{Discussion}

Clinical features

Clinical characteristics of the entire group of 111 triplenegative breast cancer patients with brain metastases
Table 5 Cox multivariate analysis of factors influencing survival from brain metastases, final model

\begin{tabular}{llll}
\hline Covariate & HR & $P$ value & $95 \%$ CI \\
\hline Karnofsky performance status & 0.319 & $<0.0001$ & $0.181 ; 0.563$ \\
$\quad$ KPS $\geq 70$ & & & \\
Age at brain metastases $>50$ & 1.681 & 0.059 & $0.981 ; 2.881$ \\
Controlled extracranial disease & 0.383 & 0.001 & $0.222 ; 0.660$ \\
\hline
\end{tabular}

confirm the results of our previous studies [2, 3] and are similar to the results of other authors $[6,8,12,21,22]$.

Due to the fact that we still do not know the biological or molecular factors that are responsible for dissemination to the brain and poor survival after the detection of brain metastases, we tried to find out if basal markers could play a major role in this processes. So we compared clinical features and survival of patients with triple-negative tumors and brain metastases which expressed and did not express basal markers. Because it was not possible to perform DNA microarray, we used IHC as a surrogate of molecular analysis. In our material, CK 5/6 was expressed in $64 \%$ of tumors. These results are in line with the findings of Nielsen et al. [21], Livacy et al. [23] and Kreike et al. [16]. HER1 was expressed in $36 \%$ of tumors and it is also in agreement with the literature; HER 1 was expressed in up to $66 \%$ of basal-like and triple-negative breast cancers, but HER1 responsible for activating gene mutations were remarkably rare [16, 21, 24-26]. The third basal marker, c-KIT was expressed in $17 \%$ of tumors. It was less than in the study by Nielsen et al. [21] and Kreike [16]. In our material, the proportion of patients with basal-like and nonbasal-like phenotypes was $76 \%$ and $24 \%$, respectively. This result was comparable to that observed by other authors [13, 14, 18, 19, 25, 27]. However, in some papers, the percentage of patients with the basal-like subtype was about 50\% [24].

There are few data in the literature concerning differences between basal-like and non-basal-like subtypes of triple-negative breast cancer according to clinical features and survival. To our knowledge, the present study is the first one analyzing such relationships in patients with brain metastases. We did not observe statistically significant differences between basal-like and non-basal-like subtypes apart from the pattern of distant metastases. Rakha et al. [13] compared the clinicopathological and immunohistochemical features of triple-negative tumors depending on the expression of specific basal markers. The authors did not show any difference in morphological features between both subtypes, but basal-like breast cancer showed distinct immunophenotypical differences with the expression of several markers of poor prognosis [13]. In addition, the authors observed a unique pattern of distant metastases of 
the basal-like subtype with more frequent metastasis to brain and lung. Such observations were similar to our observations. In the present study, in 35\% of patients with the basal-like phenotype, the brain was the first site of distant metastasis. The high propensity of the basal-like phenotype to metastasize to the brain as the first site of distant recurrence is worth further molecular investigation. It is possible that some molecular markers allow anchor cancer cells into the brain (theory of "soil and seed"). If we knew such a molecular marker, we would be able to select a group of breast cancer patients with high risk of brain metastases in order to take preventive or screening activities.

\section{Survivals}

In the present study, median DFS of all patients with triplenegative breast cancer with brain metastases was less than 2 years (20 months) and OS was less than 3 years (29 months). The results are comparable to the results by Lin et al. [22] in which median DFS of patients with triplenegative breast cancer was 19.9 months and $75 \%$ of recurrences occurred within 3 years of the diagnosis of breast cancer. Our previous study concerning 222 patients with breast cancer and brain metastases revealed statistically significant differences in median OS of patients with triple-negative, HER2-positive and ER/PR-positive HER2negative breast cancer. The differences were as follows: 2.8, 4.3 and 5.4 years, respectively, [3]. In the study by Dent et al. [4], median OS of triple-negative breast cancer was 4.2 years compared with 6 years for patients with other subtypes, and DFS was 2.6 years compared with 5 years in the other biological subtypes. In the present study, median survival from brain metastases in triplenegative breast cancer patients was 4 months. The results confirm our previous observations [2,3] and are comparable to the study done by Lin et al. [22], in which median survival time from brain metastases was 4.9 months.

The differences in survival between patients with basallike and non-basal-like breast cancer have been assessed in some studies, but the results were not unequivocal. In some of them, basal-like breast cancer patients had a significantly shorter DFS than those lacking the expression of basal markers [24, 25]. In the other studies, patients with basal-like breast cancer had significantly shorter DFS and OS times than women with other biological types of breast cancer, but basal-like status was not a significant independent prognostic variable of OS in the multivariate analysis [28, 29]. In the study by Rakha et al. [13], the difference in survival between basal-like and non-basallike subtypes was assessed in two independent cohorts of triple-negative breast cancer patients. In the first cohort of 232 patients, after the time of observation of 207 months, the basal-like subtype was associated with shorter DFS, but not OS. In the second cohort of 67 triple-negative breast cancer patients, there was no significant difference in survival between those expressing basal markers and those not expressing them.

In the present study, a very homogenous group of triplenegative breast cancer patients with brain metastases was assessed. This group had the worst prognosis out of all breast cancer patients because of the poorest biological subtype and the poorest site of dissemination. We did not show statistically significant differences in DFS, OS and survival from brain metastases which could depend on the expression of basal markers. Cox multivariate analysis revealed that the risk of death from brain metastases depends on well-known clinical features, but not on biological factors. These results are in agreement with some published data $[3,29]$. The role of KPS and the status of extracranial disease has been confirmed in our [3] and other studies [29]. It is possible that, in the group with such poor outcome, molecular markers are less important than strong clinical prognostic factors. However, the small number of patients was a serious limitation of our study and that is why our results require confirmation by other authors.

\section{Conclusions}

Triple-negative breast cancer with brain metastases is a highly selected type of cancer with very poor prognosis. The recurrence of the disease occurs within 2 years and overall survival is less than 3 years. Basal-like and nonbasal-like subgroups of patients with brain metastases did not differ significantly with regards to clinical features and survival except for the pattern of metastatic spread. Factors influencing survival from brain metastases were KPS and the status of the extracranial disease. Age at the detection of brain lesions was of borderline significance. Searching for other biological markers responsible for metastatic spread to the brain is of special value.

\section{Conflict of interests None.}

Open Access This article is distributed under the terms of the Creative Commons Attribution Noncommercial License which permits any noncommercial use, distribution, and reproduction in any medium, provided the original author(s) and source are credited.

\section{References}

1. Sorlie T, Perou CM, Tibshirani R et al (2001) Gene expression patterns of breast carcinomas distinguish tumor subclasses with clinical implications. Proc Natl Acad Sci USA 98:10869-10874

2. Niwińska A, Murawska M, Pogoda K (2010) Breast cancer subtypes and response to systemic treatment after whole-brain 
radiotherapy in patients with brain metastases. Cancer 116:4238-4247

3. Niwińska A, Murawska M, Pogoda K (2010) Breast cancer brain metastasis: differences in survival depending on biological subtype, RPA RTOG prognostic class and systemic treatment after whole brain radiotherapy (WBRT). Ann Oncol 21:942-948

4. Dent R, Trudeau M, Pritchard KI et al (2007) Triple-negative breast cancer: clinical features and patterns of recurrence. Clin Cancer Res 13:4429-4434

5. Haffty BG, Yang Q, Reiss M et al (2006) Locoregional relapse and distant metastasis in conservatively managed triple negative early-stage breast cancer. J Clin Oncol 24:5652-5657

6. Reis-Filho JS, Tutt AN (2008) Triple negative tumours: a critical review. Histopathology 52:108-118

7. Gluz O, Liedtke C, Gottschalk N, Pusztai L, Nitz U, Harbeck N (2009) Triple-negative breast cancer-current status and future directions. Ann Oncol 20:1913-1927

8. Fulford LG, Reis-Filho JS, Ryder K et al (2007) Basal-like grade III invasive ductal carcinoma of the breast; patterns of metastasis and long-term survival. Breast Cancer Res 9:R4

9. Bauer KR, Brown M, Cress RD, Parise CA, Caggiano V (2007) Descriptive analysis of estrogen receptor (ER)-negative, progesterone receptor (PR)-negative, and HER2-negative invasive breast cancer, the so-called triple-negative phenotype: a population-based study from the California cancer registry. Cancer 109:1721-1728

10. Anders CK, Carey LA (2009) Biology, metastatic patterns, and treatment of patients with triple-negative breast cancer. Clin Breast Cancer 9:73-81

11. Stockmans G, Deraedt K, Wildiers H, Moerman P, Paridaens R (2008) Triple-negative breast cancer. Curr Opin Oncol 20:614620

12. Hicks DG, Short SM, Prescott NL et al (2006) Breast cancers with brain metastases are more likely to be estrogen receptor negative, express the basal cytokeratin CK 5/6, and overexpress HER2 or EGFR. Am J Surg Pathol 30:1097-1104

13. Rakha EA, Elsheikh SE, Aleskandarany MA et al (2009) Triplenegative breast cancer: distinguishing between basal and nonbasal subtypes. Clin Cancer Res 15:2302-2310

14. Bertucci F, Finetti P, Cervera N et al (2008) How basal are triplenegative breast cancer? Int J Cancer 123:236-240

15. Carrey LA, Perou CM, Livasy CA et al (2006) Race, breast cancer subtypes, and survival in the Carolina breast cancer study. JAMA 295:2492-2502

16. Kreike B, van Kouvenhove M, Horlings H et al (2007) Gene expression profiling and histopathological characterization of triple-negative/basal-like breast carcinomas. Breast Cancer Res 9:R65

17. Prat A, Perou CM (2011) Deconstructing the molecular portraits of breast cancer. Mol Oncol 5:5-23

18. Prat A, Parker JS, Karginova O et al (2010) Phenotypic and molecular characterization of the claudin-low intrinsic subtype of breast cancer. Breast Cancer Res 12:R68

19. Hess KR, Anderson K, Symmons WF et al (2006) Pharmacogenomic predictor of sensitivity to preoperative chemotherapy with paclitaxel and fluorouracil, doxorubicin and cyclophosphamide in breast cancer. J Clin Oncol 24:4236-4244

20. Lerma E, Peiro G, Ramon T et al (2007) Immunohistochemical heterogeneity of breast carcinomas negative for estrogen receptors, progesterone receptors and Her2/neu (basal-like breast carcinomas). Mod Pathol 20:1200-1207

21. Nielsen TO, Hsu FD, Jensen K et al (2004) Immunohistochemical and clinical characterization of the basal-like subtype of invasive breast carcinoma. Clin Cancer Res 10:5367-5374

22. Lin NU, Claus E, Sohl J, Razzak AR, Arnaout A, Wiener EP (2008) Sites of distant recurrence and clinical outcomes in patients with metastatic triple-negative breast cancer: high incidence of central nervous system metastases. Cancer 113:26382645

23. Livasy CA, Karaca G, Nanda R et al (2006) Phenotypic evaluation of the basal-like subtype of invasive breast carcinoma. Mod Pathol 19:264-271

24. Rakha EA, El-Sayed ME, Green AR, Lee AH, Robertson JF, Ellis IO (2007) Prognostic markers in triple-negative breast cancer. Cancer 109:25-32

25. Tischkovitz M, Brunet JS, Begin LR et al (2007) Use of immunohistochemical markers can refine prognosis in triple negative breast cancer. BMC Cancer 7:134

26. Reis-Filho J, Pinheiro C, Lambros M et al (2006) EGFR amplification and lack of activating mutations in metaplastic breast carcinomas. J Pathol 209:445-453

27. Tan DS, Marchio C, Jones RL et al (2008) Triple-negative breast cancer: molecular profiling and prognostic impact in adjuvant anthracycline-treated patients. Breast Cancer Res Treat 111:2744

28. Banerjee S, Reis-Filho JS, Ashley S et al (2006) Basal-like breast carcinomas: clinical outcome and response to chemotherapy. J Clin Pathol 59:729-735

29. Abd El-Rehim DM, Pinder SE, Paish CE et al (2004) Expression of luminal and basal cytokeratins in human breast carcinoma. J Pathol 203:661-671 